Originalveröffentlichung in: Augst, Gerhard/Blüml, Karl/Nerius, Dieter/Sitta, Horst (Hrsg.): Zur Neuregelung der deutschen Orthographie. Begründung und Kritik. -

Tübingen: Niemeyer, 1997. S. 269-290. (Reihe Germanistische Linguistik 179)

Klaus Heller / Jürgen Scharnhorst

\title{
3.8 Kommentar zum Wörterverzeichnis
}

\section{Einleitung}

Als Grundlage für die amtliche Regelung der deutschen Orthographie, wie sie am Beginn des 20. Jahrhunderts festgelegt wurde, erschienen seinerzeit «Regeln für die deutsche Rechtschreibung nebst Wörterverzeichnis». Analog stellte sich der Internationale Arbeitskreis für Orthographie bei der Erarbeitung eines neuen Regelwerkes die Aufgabe, neben dem eigentlichen Regelteil auch einen Wörterteil vorzulegen. Dieser Entschluß war keineswegs nur durch die Tradition bedingt, sondern von der Sache her unbedingt erforderlich, stellen Regelteil und Wörterteil doch grundlegende komplementäre Bestandteile jeder orthographischen Kodifikation dar. Ihnen gegenüber würden mögliche weitere Bestandteile wie Kommentar, Beispielregister, Verzeichnis der Fachbegriffe oder gar tabellarische Darstellungen ergänzenden Charakter haben (Herberg 1993).

Während im Regelteil orthographische Sachverhalte festgestellt werden, die sich generalisieren lassen, weil sie eine mehr oder weniger große Zahl von Wörtern betreffen, findet sich im Wörterteil das einzelne Wort in seiner konkreten graphischen Form, die nur auf das jeweilige Lemma zutrifft. Wird im Regelteil jeweils nur ein bestimmtes Element oder ein bestimmter Aspekt eines Wortes angesprochen, so tritt das Wort im Wörterteil als Ganzheit auf. Die graphische Wortform ist hier sofort überschaubar, muß nicht erst aus mehreren Regeln hergeleitet werden. Die graphische Norm wird auf diese Weise von mindestens zwei Seiten ins Bewußtsein gehoben: einmal im Wörterteil, der auf die Frage antwortet «Wie schreibt man ein bestimmtes Wort?», und zum anderen im Regelteil, der darüber Auskunft gibt, welche Gesetzmäßigkeiten der Schreibung eben dieses Wortes zugrunde liegen.

Geht man von der Seite der Regeln aus, so erhebt sich die Frage, welchen Generalisierungsgrad, welche Extension eine bestimmte Regel hat. Je höher der Generalisierungsgrad, desto größer die Zahl der Wörter, die von der betreffenden Regel erfaßt werden. Je geringer der Generalisierungsgrad, um so eher ist es möglich, die Wörter, auf die die betreffende Regel zutrifft, als Beispiele aufzuführen. $\mathrm{Da}$ Vollständigkeit der Beispiele allenfalls innerhalb eines genau definierten Korpus möglich ist, liegt auf der Hand. Für den Wortschatz einer lebenden Sprache, der bekanntlich ein offenes, jederzeit veränderliches System darstellt, wird Vollständigkeit der von einer bestimmten Regel erfaßten Beispiele ohnehin ein Ideal bleiben.

Geht man vom Einzelwort aus, wie es im Wörterteil aufgeführt ist, so stellt sich die Frage, welche Regeln es denn nun sind, aus denen sich genau diese Schreibung ergibt. Dabei empfiehlt es sich, zunächst die Laut-Buchstaben-Zuordnungen zu analysieren. Untersucht man dann die syntagmatischen Beziehungen, so kommt man in die Regelbereiche für die Getrennt- und Zusammenschreibung 
und für die Schreibung mit Bindestrich. Die grammatische Analyse der Wortform führt in den Bereich der Regeln für die Groß- und Kleinschreibung. Und wiederum ein anderer Aspekt liegt vor, wenn gefragt wird, auf welchen Regeln die mögliche Worttrennung am Zeilenende beruht.

Steht die Berechtigung, ja Notwendigkeit eines Wörterteils als eines komplementären Bestandteils eines Orthographiebuches somit außer Zweifel, so warf seine Erarbeitung, die in Kooperation mit verschiedenen Experten des Internationalen Arbeitskreises für Orthographie im wesentlichen von der Arbeitsstelle für Graphie und Orthographie (Wolfgang Mentrup, Klaus Heller) am Institut für deutsche Sprache in Mannheim vorgenommen wurde, eine Reihe von Fragen auf, die mit der Spezifik eines solchen Wörterverzeichnisses zusammenhängen. Im Folgenden sollen einige dieser Probleme erörtert werden.

Da das Wörterverzeichnis Teil eines amtlichen Regelwerks ist, können nicht die Anforderungen, die breite Benutzerkreise üblicherweise an orthographische Nachschlagewerke stellen, bestimmend sein. Das hat Auswirkungen sowohl auf die Auswahl der in das Verzeichnis aufzunehmenden Stichwörter als auch auf die Gestaltung der Einträge selbst. In beiden Fällen gilt als Grundsatz: Konzentration auf das orthographisch Relevante. Zu Recht erwartet der Benutzer eines amtlichen Wörterverzeichnisses Auskunft über die Schreibung eines jeden wichtigen Wortes der deutschen Standardsprache, selbst wenn sich diese aus den Regeln folgerichtig ergibt. Der Wörterteil ist zwar nicht für den unmittelbaren Gebrauch etwa in der Schule gedacht, stellt aber zusammen mit dem Regelteil die Grundlage für die Schreibung der Behörden und der Schulen dar, ist sozusagen das Urmeter der deutschen Orthographie. Nach Meinung seiner Bearbeiter mußte es daher als unbefriedigend angesehen werden, für den Wörterteil des neuen amtlichen Regelwerks eine so zufällige Auswahl zu treffen, wie das z. B. bei den preußischen Regelwerken (Regeln 1880 und Regeln 1902) der Fall war. Sie stellten sich daher die Aufgabe, ein Verzeichnis zu erarbeiten, das alle dem Zentrum der gegenwärtigen deutschen Standardsprache zuzurechnenden Morpheme in wenigstens einem Wortbeispiel enthält und darüber hinaus Wortbeispiele aus verschiedenen Sektoren der Peripherie anbietet.

\section{Das Konzept von Zentrum und Peripherie und seine Anwendung}

\subsection{Zentrum und Peripherie als linguistische Universalien}

Das Konzept von Zentrum und Peripherie, das vor allem von Linguisten der Prager Schule entwickelt und ausgearbeitet worden ist (vgl. Travaux 1966; Daneš 1982; Heller/Scharnhorst 1983), beruht auf dem Gedanken, daß die Sprache nicht schlechthin ein System ist, sondern daß sie ein dynamisches System ist. Alle Veränderungen, die im sprachlichen Zeichensystem eintreten und dabei selbstverständlich auf Sprachhandlungen der Mitglieder der betreffenden Sprachgemeinschaft zurückgehen, sind Ausdruck der elastischen Stabilität des Systems. Bei der Behandlung diachronischer Fragen läßt sich zeigen, wie bestimmte Elemente aus anderen Systemen in ein gegebenes System eindringen, also dessen "Grenze» überschreiten, zunächst an der Peripherie verharren, um dann möglicherweise die «Übergangssphäre» zu erreichen und unter günstigen Umständen 
vielleicht das «Zentrum» zu bereichern. Solche Prozesse können sich über Jahrhunderte hinziehen, sie können aber auch in wesentlich kürzeren Zeiträumen vor sich gehen. Daneben finden solche Bewegungen auch in umgekehrter Richtung, also vom Zentrum zur Peripherie, statt.

Was die synchronische Betrachtung des Systems angeht, so muß der Relation von Zentrum und Peripherie der Rang eines universalen Merkmals der Sprache zuerkannt werden. Als generelles Kriterium für die Zuordnung eines sprachlichen Elements zu Zentrum, Übergangssphäre oder Peripherie wird in erster Linie der Grad der Eingliederung in das zur Rede stehende System gesehen, gleich welcher Art dieses auch ist. Dabei gilt, $\mathrm{da} \beta$ zentrale Erscheinungen ausschließlich oder vorwiegend durch Merkmale charakterisiert sind, die für das betreffende System konstitutiv sind, während periphere Erscheinungen solche Merkmale in geringerem Maße aufweisen, aber statt dessen mehr oder minder Merkmale anderer Systeme enthalten. Es versteht sich dabei von selbst, daß die Kriterien im einzelnen für jede Ebene des sprachlichen Zeichensystems und jeden Gegenstand der Betrachtung speziell ausgearbeitet werden müssen. Für den Sprachgebrauch gilt, $\mathrm{da} \beta$ zentrale Erscheinungen in der Regel häufiger vorkommen als periphere. Auch das Vorhandensein von Varianten weist auf Randerscheinungen hin.

\subsection{Kriterien für die Zuordnung von Lexemen zu Zentrum und Peripherie}

Für unseren Zusammenhang ist der Blick vor allem auf die morphematische und die lexikalische Ebene und auf deren Überschneidungsbereich mit der graphischen Ebene zu richten (zum zugrunde gelegten Ebenenmodell vgl. Nerius / Scharnhorst 1980; Nerius et al. 1989).

Mit den Kriterien, die für die Zuordnung eines Wortes zum Zentrum oder zur Peripherie des als System aufgefaßten Wortschatzes wesentlich sind, hat sich Filipec (1966) beschäftigt. Dabei sind für uns die folgenden Überlegungen wichtig:

1) Ist die Wortform als heimisch oder als fremd einzuordnen?

Dabei ist einerseits die graphische Wortform, andererseits die phonische Wortform sowie drittens die Beziehung zwischen beiden zu analysieren. Heimisch-deutsche Wörter (genauer: nichtentlehnte Wörter mit entsprechender Phonem- und Graphemstruktur) gehören zum Zentrum, Fremdwörter dagegen zur Peripherie. Lehnwörter aber (also Wörter, die ursprünglich aus einer fremden Sprache entlehnt wurden, sich in ihrer Formstruktur aber der Struktur heimischer Wörter angeglichen haben) tendieren zum Zentrum.

2) Wie ist die Morphemstruktur des Wortes?

Handelt es sich um monomorphematische Wörter, in denen Basismorphem und Wort identisch sind, oder um bi- oder polymorphematische Wörter? Liegt ein Simplex, ein Kompositum oder eine Derivation (eine Präfix- oder Suffixbildung) vor?

3) Welche stilistische Charakteristik hat das Wort?

Während stilistisch neutrale Wörter zum Zentrum gehören, sind stilistisch markierte Wörter der Peripherie zuzuordnen. 
4) Gehört das Wort zu einer Fachsprache, ist es ein Terminus oder Professionalismus?

Das Zentrum wird selbstverständlich von den Wörtern gebildet, die allgemeinsprachlich sind, nicht an eine bestimmte Terminologie oder einen Beruf gebunden sind, während spezielle Fachwörter, Termini und Professionalismen die Peripherie bilden.

5) Welchen Platz nimmt das betreffende Wort auf der Zeitachse ein?

Wenn das Wort veraltet ist, liegt es an oder außerhalb der Grenze eines synchron-gegenwartssprachlich determinierten Systems. Neologismen und veraltende Wörter bewegen sich dagegen meist an der Peripherie, während die zeitlich nicht markierten Wörter das Zentrum bilden. Historismen tendieren zur Peripherie.

6) Ist das Wort in seiner territorialen Geltung eingeschränkt, oder wird es im gesamten Gebiet der betreffenden Sprache gebraucht?

7) Handelt es sich um ein Appellativum oder um einen Eigennamen?

Es ist klar, daß Eigennamen nur in Ausnahmefällen einen Platz im Zentrum einnehmen, meist dann, wenn sie ihre propriale Bedeutung aufgegeben haben.

8) Wie steht es mit der Frequenz des Wortes?

Häufig gebrauchte Wörter tendieren zum Zentrum, selten gebrauchte hingegen zur Peripherie.

Stellt man sich vor, der Wortschatz einer Sprache sei auf einer kreisförmigen Fläche angeordnet und die Nähe eines bestimmten Wortes zum Mittelpunkt des Kreises bzw. seine Entfernung von ihm sei durch die oben genannten Kriterien bestimmt, so müssen die Stellung im Innern des Zentrums solche Appellativa einnehmen, die heimischer Herkunft, monomorphematisch, häufig gebraucht und stilistisch neutral sind, die als allgemeinsprachlich (also nicht eng fachsprachlich) gelten, weder veraltet noch veraltend und im gesamten Verbreitungsgebiet der betreffenden Sprache üblich sind. Bei vielen Wörtern werden bestimmte Kriterien auf einen Platz im Zentrum, andere in Richtung Peripherie weisen. Hier hängt die Einordnung vom Abwägen der verschiedenen Kriterien, von ihrer $\mathrm{Ge}$ wichtung ab. Wesentlich für das Konzept von Zentrum und Peripherie ist, daß das Areal zwischen Mittelpunkt und Grenze als Kontinuum gedacht ist, so daß Entscheidungen nicht auf ein Entweder-Oder, sondern auf ein Mehr-oder-Weniger hinauslaufen. Das Konzept von Zentrum und Peripherie ist ein gedankliches Modell, das bei der Lösung vieler Probleme der Sprachbeschreibung und der Kodifizierung von Normen hilfreich sein kann, so auch bei der Stichwortauswahl für das Wörterverzeichnis eines orthographischen Regelwerkes.

\subsection{Die Auswahl der Stichwörter}

Die Aufgabe bei der Erarbeitung eines Vorschlags für das Wörterverzeichnis war es demnach, bei der Auswahl der Stichwörter nicht willkürlich zu verfahren, sondern unter dem Gesichtspunkt von Zentrum und Peripherie möglichst systematisch vorzugehen. Daß hierbei keine scharfen Grenzen gezogen werden konnten, war von vornherein klar. Jedes der im Folgenden anzusprechenden Kriterien zeigt einen mehr oder minder deutlichen Übergangsbereich zwischen Zentrum und Pe- 
ripherie, der von Sprachteilhaber zu Sprachteilhaber je nach landschaftlicher Gebundenheit, Alter, sozialer Zugehörigkeit, Beruf, Bildungsgrad, spezieller Interessenlage usw. anders gesehen wird. Im allgemeinen sind aber - mit der Einschränkung, daß orthographische Relevanz diese Auswahlkriterien gelegentlich dominieren kann - folgende Gruppen von Lexemen in das Wörterverzeichnis aufgenommen bzw. nicht aufgenommen worden:

1) Aufgenommen wurden heimische Wörter (einschließlich der Lehnwörter) und übliche Fremdwörter. Nicht aufgenommen wurden dagegen seltene Fremdwörter, die nur der Bildungssprache angehören (z. B. Egghead, Entourage, Miszellen, Phantasmagorie, Rabulistik, voluptuös), sowie fremdsprachliche Wendungen und Zitatwörter (z. B. all right!, corriger la fortune, de facto, dolce far niente, Enfant terrible), zu denen z. B. auch die zahlreichen Tempound Vortragsbezeichnungen in der Musik (allegro, con brio usw.) zählen, die allenfalls noch substantivisch als Satzbezeichnungen akzeptiert worden sind (Allegro, Largo usw.). Dagegen wurde üblichen Fremdwörtern, auch wenn sie im Sprachgebrauch nicht gerade häufig auftreten, in reichem $\mathrm{Maße} \mathrm{Zu}$ gang in das Wörterverzeichnis gewährt.

2) Komposita, deren Glieder als Simplex an alphabetischer Stelle verzeichnet sind (z. B. Aalreuse, Postbote, Rahmenplan, Showgeschäft) wurden nicht aufgenommen; desgleichen sind nicht verzeichnet reguläre Derivationen mit Suffixen (z. B. altern, harmonieren, kühlen, rasten; freundlich, sparsam, tragbar, trostlos, windig; Altertum, Fischer, Fischerei, Freundin, Kühlung, Sparsamkeit, Wildnis, Wissenschaft) und Präfixen (z. B. beeilen, entrosten, verlaufen; interkontinental, unfreundlich; Disharmonie, Missgeburt, Unwetter). Man vergleiche dazu Augst (1975).

3) Die meisten Wörter, die der saloppen, der derben und der vulgären Stilschicht zuzuordnen sind, wurden nicht aufgenommen. Das gleiche trifft auf viele Wörter der dichterischen Stilschicht zu (z. B. Odem), wohingegen Aar wegen der doppelten Vokalbuchstaben im Wörterverzeichnis steht. Dagegen fand eine größere Zahl umgangssprachlicher Wörter, die heute in der nichtoffiziellen schriftlichen Kommunikation häufiger auftreten (z. B. bummeln, flunkern, kriegen, Fimmel, Fisimatenten, Stups, verdattert), Eingang in das Wörterverzeichnis.

4) Ähnlich problematisch ist die Ausgrenzung fachsprachlicher Bezeichnungen, und zwar nicht nur wegen der vielfältigen Übergänge zwischen fachsprachlichem und nichtfachsprachlichem Gebrauch, zwischen Allgemeinverständlichkeit und Nichtallgemeinverständlichkeit, zwischen Terminus und allgemeinsprachlicher Bezeichnung (vgl. Heller 1970). Wie überhaupt in den Randbereichen des Allgemeinwortschatzes, so treten auch hier verstärkt orthographische Probleme auf, so daß sich die Bearbeiter in einem ständigen Konflikt zwischen dem Streben nach größtmöglicher Straffung und orthographisch bedingter Ausweitung befanden. So ist z. B. auf fachspezifische Schreibungen nicht verzichtet worden, doch wird entsprechend verwiesen (z. B. Calcit s. Kalzit; Kalzit, fachspr. Calcit usw.)

5) Nicht immer klar entschieden werden konnte im Hinblick auf veraltetes Wortgut. So wurden Gevatter und Oheim nicht aufgenommen, während $\mathrm{Ha}$ gestolz und Jungfer (wegen Jungfernfahrt) Eingang ins Wörterverzeichnis 
fanden. Aufgenommen wurde auch eine Reihe von Historismen, d. h. solche Bezeichnungen, die einen geschichtlichen Bezug haben und heute noch im entsprechenden Zusammenhang verwendet werden (z. B. Galeere, Hellebarde, Kemenate).

6) Regionale und mundartliche Wörter wurden nicht aufgenommen. Länderspezifische Wörter (Austriazismen und Helvetismen) werden jedoch verzeichnet, sofern sie in Österreich bzw. in der Schweiz als standardsprachlich gelten. Sie sind im Wörterverzeichnis wie alle anderen Wörter des deutschen Wortschatzes behandelt, also nicht markiert (z. B. Beugel, Faktura). Ihre Auswahl oblag den österreichischen und Schweizer Experten. Handelt es sich jedoch um österreichische und Schweizer orthographische Varianten, so werden sie entsprechend gekennzeichnet, z. Bsp. Gulasch, (österr. auch) Gulyás; Küken, Kücken (österr.); Cervelat (schweiz.), auch Servela (schweiz.).

7) Ausgeklammert wurden schließlich alle Eigennamen; jedoch finden sie sich, wenn sie wie Adonis zu Appellativa geworden sind, was gelegentlich auch für Ableitungen (sibyllinisch) und für Zusammensetzungen (Rhesusfaktor, Schweizergarde) gilt.

\subsection{Empirische Untersuchungen}

Um die Auswahlprinzipien für das Wörterverzeichnis zu veranschaulichen, geben wir im folgenden Ergebnisse einer empirischen Untersuchung (vgl. Scharnhorst 1991 u. 1993) wieder, die an einem bestimmten Abschnitt des Alphabets (los bis Lost generation) zeigt, wie auf Grund der oben genannten Kriterien die Zuordnung zu Zentrum und Peripherie im einzelnen vorgenommen wurde. Die folgende Liste, zu der das WDG (1964-1977) sowie Augst (1975), Duden: Deutsches Universalwörterbuch (1989), Österreichisches Wörterbuch (1990) und Pfeifer (1995) zu Rate gezogen wurden, basiert hauptsächlich auf Duden-Rechtschreibung (1991):

\begin{tabular}{|c|c|c|c|c|}
\hline Zentrum & Übergangssphäre & Peripherie & Grenze & $\begin{array}{l}\text { andere } \\
\text { Systeme }\end{array}$ \\
\hline $\begin{array}{ll}\operatorname{los} & \\
& \operatorname{los} \cup\end{array}$ & $\begin{array}{l}\text { los [sein] } \\
\text {...bekommen } \\
\text {...binden } \\
\text {...brechen } \\
\text {...fahren } \\
\text {...gehen } \\
\text {...kaufen } \\
\text {...kommen } \\
\text {...lassen } \\
\text {...laufen } \\
\text {...lösen } \\
\text {...machen } \\
\text {...marschieren } \\
\text {...reißen }\end{array}$ & $\begin{array}{l}\text { Los-von-Rom- } \\
\text { Bewegung } \\
\text {...brausen (ugs.) } \\
\text {...eisen (ugs.) } \\
\text {...haben (ugs.) } \\
\text {...heulen (ugs.) } \\
\text {...kriegen (ugs.) } \\
\text {...legen (ugs.) } \\
\text {...schießen (ugs.) } \\
\text {...stürzen (ugs.) } \\
\text {...werden (ugs.) } \\
\text {...ziehen (ugs.) }\end{array}$ & & \\
\hline
\end{tabular}




\begin{tabular}{|c|c|c|c|c|}
\hline Zentrum & Übergangssphäre & Peripherie & Grenze & $\begin{array}{l}\text { andere } \\
\text { Systeme }\end{array}$ \\
\hline $\begin{array}{l}{ }^{1} \text { Losung } \\
\text { lose }\end{array}$ & $\begin{array}{l}\text {...sagen } \\
\ldots \text {..schicken } \\
\text {...schlagen } \\
\text {...schrauben } \\
\ldots \text {.sprechen } \\
\text {...steuern } \\
\text {-los (z. B. } \\
\text { arbeitslos) } \\
\text { das große Los } \\
\text { Los } \cup\end{array}$ & 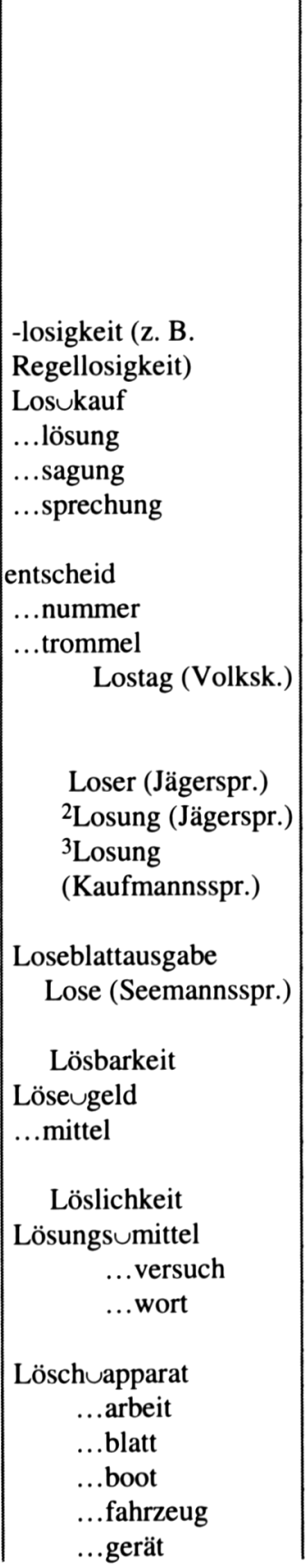 & $\begin{array}{l}\text { 2losen } \\
\text { 〈horchen〉 }\end{array}$ & $\begin{array}{l}\text { 2löschen } \\
\text { (erlöschen> } \\
\text { (veralt.) }\end{array}$ \\
\hline
\end{tabular}




\begin{tabular}{|c|c|c|c|c|}
\hline Zentrum & Übergangssphäre & Peripherie & Grenze & $\begin{array}{l}\text { andere } \\
\text { Systeme }\end{array}$ \\
\hline & $\begin{array}{l}\text { Löscher } \\
\text { 33öschen [Ladung] } \\
\text { Löß (Geol.) }\end{array}$ & $\begin{array}{l}\quad \text {...wasser } \\
\text {...zug } \\
\text { Löschukalk } \\
\text { Löschutaste } \\
\text { Löschung } \\
\text { Lößuboden } \\
\text {...landschaft } \\
\text {...schicht (Geol.) } \\
\text { lössig (Geol.) } \\
\text { Lößukindel } \\
\text { (Geol.) } \\
\text { Lost (Milit.) } \\
\quad \text { Löl } \\
\quad \text { Lost }\end{array}$ & $\begin{array}{c} \\
\\
\\
\text { hitz (EN) } \\
\text { Los Ang } \\
\text { generation }\end{array}$ & eles (EN) \\
\hline
\end{tabular}

Die Zuordnung des Adverbs los zum Zentrum bedarf keiner Erörterung. Es handelt sich um ein Basismorphem, das - aus dem Germanischen übernommen - bereits seit althochdeutscher Zeit zum heimischen Wortschatz gehört. Aus dem adverbiellen Gebrauch hervorgegangen, ist los- seit dem Frühneuhochdeutschen als erstes Glied in Verbalkomposita nachweisbar und - wie die in unserer Liste aufgeführten zahlreichen Bildungen mit los- zeigen - äußerst produktiv. Die Wörterbücher geben für einen Teil dieser Verbalkomposita im Zusammenhang mit ihrer speziellen Semantik als Stilschicht «umgangssprachlich» an, weshalb sie entsprechend den oben dargelegten Kriterien hier der Peripherie zugeordnet werden. Für die Aufnahme in das Wörterverzeichnis kommen die Verbalkomposita mit los- im einzelnen nicht in Frage, da ihre Basismorpheme jeweils an alphabetischer Stelle aufgeführt werden. So genügt es, wenn sie hier als Wortbildungstyp vertreten sind, was dadurch geschieht, daß das erste Kompositionsglied mit einem Verb als Beispiel angeführt wird, nach dessen Muster weitere Bildungen möglich sind: losubinden... (der Bogen gibt in Verbindung mit den drei nachgestellten Punkten an, daß noch weitere Wörter anstelle des genannten angeschlossen werden können).

Ähnlich, aber doch wieder anders liegt der Fall bei -los, das zwar ebenfalls seit althochdeutscher Zeit im Deutschen heimisch ist (ahd. brôtelôs hungrig)), sich aber von einem ursprünglich zweiten Kompositionsglied bei Adjektiven zu einem Adjektivsuffix entwickelt hat. Aus linguistischer Sicht wäre es naheliegend, ein solches produktives Wortbildungsmorphem (z. B. ahnungs-, atem-, brot-, charakter-, disziplin-, farb-, frucht-, gefühlslos usw.) mit in das Wörterverzeichnis aufzunehmen. Außerlinguistische Gründe, die einmal mit der Umfangsbegrenzung des Wörterverzeichnisses, zum anderen aber mit dessen amtlichem Charakter zusammenhängen, zwangen in diesem wie in ähnlichen Fällen leider dazu, auf die Eintragung ins Wörterverzeichnis zu verzichten. 
Unzweifelhaft ist auch die Zuordnung des Substantivs Los «Schicksal, Lotterieschein> zum Zentrum. Auch hier handelt es sich um ein altes heimisches Wort, um ein Verbalabstraktum zu einem im Neuhochdeutschen untergegangenen starken Verb ahd. (h)lioßan 〈losen`, das die Sitte der Germanen bezeichnete, aus der Lage geworfener Runenstäbe zu weissagen. Das Verb ${ }^{1}$ losen (mit dem Exponenten versehen, um es von ${ }^{2}$ losen zu unterscheiden) ist eine schwach flektierende Neubildung zum Substantiv Los. Da es orthographisch keine Besonderheiten bietet, wurde es nicht in das Wörterverzeichnis aufgenommen.

Aus einem ganz anderen Grunde wurde 2 losen 〈horchen, zuhören〉 nicht aufgenommen. Das Wort hat etymologisch mit ${ }^{1}$ losen nichts zu tun, sondern leitet sich von einem ahd. Verb (h)losên her. Im 16. und auch noch im 17. Jahrhundert war es in der Schriftsprache verbreitet, jedoch nur im Oberdeutschen. Seitdem lebt es nur noch in den süddeutschen, österreichischen und schweizerischen Mundarten, gehört also nicht der gegenwärtigen Standardsprache an (vgl. DWB: 6, 1188). Das von diesem Verb abgeleitete Substantiv Loser Lauscher, Ohr beim Schalenwild (außer Wildschwein), bei Wolf, Fuchs und Biber` gehört als Wort der Jägersprache der Peripherie zu und wurde ebenfalls nicht ins Wörterverzeichnis aufgenommen.

Unter der Schreibung Losung, die sich im Wörterverzeichnis findet, sind drei Homonyme mit unterschiedlicher Etymologie, Bedeutung und Gebrauchssphäre subsumiert. 'Losung (Leitwort, Parole, Wahlspruch) ist in dieser Bedeutung seit dem 16. Jh. bezeugt und eine Ableitung von Los, das in frühneuhochdeutscher Zeit auch (Erkennungszeichen, Parole) bedeutete. Hingegen ist ${ }^{2}$ Losung eine in der Jägersprache übliche Bezeichnung für den 〈Kot vom Wild und vom Hund〉, eine Ableitung zu dem veralteten weidmännischen losen (den Kot loslassen), das etymologisch zu los gestellt wird. Davon abzusetzen ist ${ }^{3}$ Losung als Terminus der Kaufmannssprache in der Bedeutung <Tages-, Kasseneinnahme in einem Einzelhandelsgeschäft oder Kaufhaus'; es leitet sich von lösen in der veralteten $\mathrm{Be}$ deutung «als Erlös einbringen ’ her.

Das Adjektiv lose locker», ursprünglich im Mittelhochdeutschen als Adverb zu lôs gebildet, dann aber semantisch und formal davon getrennt, ist als Basismorphem dem Zentrum zuzuweisen und deshalb ins Wörterverzeichnis aufgenommen. Dagegen wurde das vom Adjektiv lose gebildete Substantiv die Lose, ein Wort der Seemannssprache mit der Bedeutung «schlaffes, durchhängendes Tau(stück), nicht aufgeführt.

Obwohl zur Peripherie gehörend, fand Loseblattausgabe wegen seines besonderen Wortbildungscharakters als Kompositum mit Zusammenschreibung - es handelt sich nicht um eine lose Blattausgabe, sondern um eine Ausgabe [in Form] loser Blätter, d. h. um eine Veröffentlichung, die in einzelnen Blättern erscheint Eingang in das Wörterverzeichnis. Das gleiche wäre für das Kompositum Losvon-Rom-Bewegung im Prinzip möglich gewesen. Dieser Typ mit BindestrichSchreibung ist im Regelteil $\S 43$ durch andere Beispiele vertreten (z. B. das Aufdie-lange-Bank-Schieben, das An-den-Haaren-Herbeiziehen).

Das Verb lösen (so schon mittelhochdeutsch als Ableitung zum Adjektiv lôs) ist wegen der Umlautschreibung im Wörterverzeichnis vertreten. Dagegen sind die Ableitungen lösbar, löslich einschließlich ihrer Derivationen nicht ins Wörterverzeichnis eingegangen. 
Über die Zuordnung von ${ }^{1}$ löschen (im Mhd. noch in der Form leschen) zum Zentrum dürfte kein Zweifel bestehen, wenn es sich um das transitive Verb mit solchen Objekten wie Brand, Feuer handelt. Dagegen ist das intransitive Verb 2 löschen (die Flamme, das Leben lischt, Prät. losch) veraltet und bleibt folglich außerhalb des Wörterverzeichnisses. Mit der Schreibung von ${ }^{1}$ löschen identisch ist die von ${ }^{3}$ löschen (Frachtgüter ausladen, Schiffe entladen), das der Seemannssprache entstammt und aus mnd. lössen 〈leer, leicht machen〉 (einer Bildung zu los) unter lautlicher Angleichung an 1 löschen ins Hochdeutsche übernommen wurde. Unter der Schreibung löschen sind im Wörterverzeichnis also zwei Homonyme vereint.

Über die zu löschen gehörenden Ableitungen und Zusammensetzungen ist Entsprechendes zu sagen wie oben bei lösen.

$L \ddot{\beta} \beta$, die Bezeichnung für kalkhaltigen Lehmstaub als Ablagerung von Eiszeitwinden, ist ein ursprünglich geologischer Begriff, der sich vermutlich an alemannisch lösch «locker> (wohl eine mundartliche Form von los, lose) anlehnt. Seit 1823, als dieses Wort von dem Geologen Karl Cäsar v. Leonhardt auf Grund seiner Untersuchungen am alemannischen Oberrhein geprägt wurde, hat es sich über den Kreis der Fachleute hinaus - auch in Form von Komposita - verbreitet. Deshalb haben wir es der Übergangssphäre zwischen Zentrum und Peripherie zugewiesen und in das Wörterverzeichnis mit aufgenommen.

Dagegen blieb Lost, die militärische Tarnbezeichnung eines Kampfstoffes für den Gaskrieg, die nach den Chemikern Lommel und Steinkopff benannt wurde, unberücksichtigt.

Entsprechend dem Grundsatz, daß Eigennamen nicht aufgenommen werden, erhielten Lößnitz (der Name einer Landschaft nordwestlich von Dresden) und Los Angeles keinen Eingang in das Wörterverzeichnis.

Die gleiche Entscheidung wurde hinsichtlich des Fremdlexems Lost generation, einer Bezeichnung für die ‘verlorene Generation` nach dem ersten Weltkrieg, getroffen. Es handelt sich um eine aus dem Englischen übernommene, im Deutschen selten gebrauchte Wortverbindung mit Grenzcharakter am Rande der Peripherie.

Die Bearbeiter des Wörterverzeichnisses stellten sich eine doppelte Aufgabe: ein Verzeichnis zu erarbeiten, das (a) alle dem Zentrum der gegenwärtigen deutschen Standardsprache zuzurechnenden Morpheme in wenigstens einem Wortbeispiel enthält und (b) darüber hinaus typische Wortbeispiele aus verschiedenen Sektoren der Peripherie bietet. Das Wörterverzeichnis selbst sagt direkt nichts darüber aus, welche Stellung das einzelne Stichwort im Spannungsfeld von Zentrum und Peripherie einnimmt, wie der folgende kleine Ausschnitt zeigt, der das zusammenfaßt, was im Vorhergehenden besprochen wurde:

$\begin{array}{ll}\text { los } & \text { Loseblattausgabe } \\ \text { Los } & \text { lösen } \\ \text { losubinden } & \text { Löß } \\ \text { löschen } & \text { Losung } \\ \text { lose } & \end{array}$

Auf die Systematik der Wortbildung gerichtete Überlegungen und Analysen führten also zu einer Reihe von Ableitungsmodellen und erlaubten es schließlich, 
strukturelle Grundtypen zu ermitteln, die vorrangig für eine Aufnahme in das Wörterverzeichnis in Frage kamen. Im allgemeinen konnte dabei dem Grundsatz gefolgt werden, Ableitungen und Zusammensetzungen nur dann aufzunehmen, wenn das in orthographischer Hinsicht gerechtfertigt ist.

In ähnlicher Weise konnte eine Übersicht über die Umlautprofile gewonnen werden (Umlaut im Plural, in Verbformen, bei Komparation, in Diminutivformen, bei Movierung und sonstigen Ableitungen) sowie über den Ablaut und den $e / i$ Wechsel bei Verben. Auch die Besonderheiten bei Fremdwörtern, z. B. die Pluralbildung (Appendix - Appendices) oder das Alternieren der Stammschreibung (annektieren - Annexion, applaudieren - Applaus, absorbieren - Absorption, auch Job-jobben) wurden unter orthographischem Gesichtspunkt untersucht. Es wurde damit möglich, hier Entscheidungen zu fällen, welche die Behandlung von Fallgruppen und nicht Einzelbeispiele betreffen, sich also stets auf systematische Gesichtspunkte zurückführen lassen.

\section{Zur Makro- und Mikrostruktur des Wörterverzeichnisses}

\subsection{Zur Makrostruktur}

Als Ergebnis des langwierigen Auswahlprozesses liegt ein Wörterverzeichnis vor, das etwa 12'500 Stichwörter umfaßt. Zum Vergleich: Das amtliche Wörterverzeichnis von 1902 enthielt ca. 6'200 Stichwörter; die darauf basierende 7. Auflage von Konrad Dudens orthographischem Wörterbuch verzeichnete etwa 32’000 Stichwörter. Demgegenüber enthält die 20. Auflage des Rechtschreibdudens von 1991 rund 115'000 Stichwörter.

Die Anordnung der ca. 12'500 Stichwörter im Wörterverzeichnis erfolgt «striktalphabetisch» (Wiegand 1989a: 380ff.). Die «Umlautbuchstaben» $\ddot{a}, \ddot{o}$, $\ddot{u}$ werden dabei unmittelbar jeweils nach den Buchstaben $a, o, u$ eingeordnet, also bei der alphabetischen Einordnung nicht in $a e, o e$, ue aufgelöst. Es steht also nützen unmittelbar hinter nutzen und nicht etwa zwischen Nudist und Nugat. Der Buchstabe $\beta$ wird wie ss eingeordnet, also Buße zwischen Bussard und Busserl.

\subsection{Zur Mikrostruktur}

Ebenso wie bei der Makrostruktur gilt auch für die Mikrostruktur: Konzentration auf das orthographisch Relevante. Im Normalfall bedeutet das, daß das Lexem in seiner graphischen Form ohne alle weiteren Zusätze aufgeführt wird. Grundsätzlich wird also darauf verzichtet, Angaben zu machen, die keinen Bezug zur Schreibung haben, sondern die Aussprache, die Flexion, die Semantik sowie stilistische, regionale oder fachsprachliche Markierungen betreffen.

\subsubsection{Homonyme, Homophone, Homographe}

Wie oben bereits dargelegt, wird auch auf die Unterscheidung von Homonymen verzichtet. Davon gibt es jedoch einige Ausnahmen:

Im Fall von Homophonen, also gleich gesprochenen Wörtern, die unterschiedlich geschrieben werden, wird auf die Bedeutung zurückgegriffen, z. B. Saite (beim 
Musikinstrument) : Seite (etwa im Buch). Außerdem wird unter dem Zeichen $\neq$ wechselseitig von einem Stichwort auf das andere verwiesen.

Parallel dazu wird im Fall von Homographen, also gleich geschriebenen Wörtern, die unterschiedlich ausgesprochen werden, ebenfalls ein Hinweis auf die Bedeutung gegeben, jedoch nur im Sinne einer Identifikationsangabe, z. B. Band (zu binden) und Band (Musikgruppe).

Besondere Aufmerksamkeit forderten Wörter, die in Schreibung und/oder Aussprache und/oder Bedeutung so ähnlich sind, daß sie leicht verwechselt werden. Hier wurde meist ähnlich verfahren wie bei den Homophonen, z. B. Akzidens (Zufälliges) $\neq$ Akzidenz (Druckwesen); Apartment (kleine Wohnung) $\neq$ Appartement (Zimmerflucht im Hotel). Hinzugefügt sind wechselseitige Verweise von einem Stichwort auf das andere.

Wie oben bereits am Beispiel von los, Los gezeigt, erhalten unterschiedliche Wortarten getrennte Einträge ohne Kommentar (z. B. auch bar, Bar; wagen, Wagen).

\subsubsection{Orthographische und andere Varianten}

Für ein orthographisches Wörterverzeichnis wichtig ist die Frage der Varianten. Dabei kommt es auf die speziell orthographischen Varianten an, die von Gabler (1992: 376) definiert werden als

Graphemfolgen, die zur Materialisierung ein und derselben Bedeutung (eines Morphems, eines Wortes, eines Lexems, eines Satzes) in der geschriebenen Sprache dienen. Diese Graphemfolgen unterscheiden sich in bezug auf ein oder mehrere Grapheme, beziehen sich aber auf dieselbe Phonemfolge. Oder kurz: Orthographische Varianten sind unterschiedliche Schreibungen einer Bedeutung bei gleicher Lautung.

Demnach können Aussprachevarianten oder morphematische Varianten (z. B. Ellbogen, Ellenbogen; Funke, Funken; Gleis, Geleise) nicht als orthographische Varianten angesehen werden. Sie werden jedoch beide angeführt, wenn sie in der Standardsprache üblich sind. Von praktischer Bedeutung ist die Unterscheidung von orthographischen und anderen Varianten insofern, als nur bei ersteren zwischen Haupt- und Nebenvariante differenziert wird. Sofern sich eine orthographische Hauptvariante (im Sinne einer empfohlenen, zu bevorzugenden Schreibung) und eine Nebenvariante (im Sinne einer auch vorhandenen, möglichen Schreibung) unterscheiden lassen, wird von der Nebenvariante durch "siehe» auf die Hauptvariante verwiesen, z. B. Anchovis s. Anschovis, während bei der Hauptvariante die Nebenvariante nur genannt wird: Anschovis, auch Anchovis. Eine solche Variantenführung ist besonders bei Doppelschreibungen angebracht, die eine Folge integrierter Fremdwortschreibung sind, z. B. Bouclé, auch Buklee und Buklee s. Bouclé. Dem Wesen der allmählichen, über längere Zeiträume vor sich gehenden Integration von Fremdwörtern entsprechend (vgl. Heller 1980; Heller/Walz 1992), müssen diese Varianten behutsam «individuell» geführt werden, dürfen nicht über einen Kamm geschert werden. Während bei einer älteren, längst üblichen eingebürgerten Schreibung unter Umständen auf die ursprüngliche, der Herkunftssprache nahe Schreibung ganz verzichtet werden kann, wird sie in anderen Fällen als Nebenform noch erhalten bleiben, wohingegen jüngere Integrationsschreibungen und insbesondere aktuelle Reformschreibungen, sofern sie sich nicht an ein vorhandenes Muster anschließen lassen, in der Regel nicht die Hauptform darstellen. 
Zu beachten ist, daß in der Schweiz, aber auch in Österreich manche der im Wörterverzeichnis als Nebenform angegebenen Fremdwortschreibungen gebräuchlicher sind als im übrigen deutschen Sprachraum. Daß österreichische und Schweizer orthographische Varianten als solche besonders gekennzeichnet werden, wurde bereits oben erwähnt. Grundsätzlich werden deutsche, österreichische und schweizerische Schreibungen als gleichberechtigt angesehen, so daß es hier keine Kennzeichnung als Haupt- oder Nebenvariante gibt.

Die nichtorthographischen Varianten werden in jedem Falle streng alphabetisch aufgeführt, und zwar auch dann, wenn dadurch die Hauptform erst an zweiter Stelle genannt wird, z. B. Heuschreck, Heuschrecke; Strieme, Striemen. Eine Variantenführung in solchen Fällen kann nicht Sache eines orthographischen Regelwerkes sein. Grenzfälle gibt es jedoch auch hier, vor allem dann, wenn die Aussprache schwankt oder sich nur geringfügig unterscheidet, so daß man versucht ist, orthographische Varianten anzusetzen. Man vergleiche dazu Muthmann (1994: 7):

So einleuchtend und praktikabel die Großeinteilung in Aussprachevarianten, orthographische Varianten und Formvarianten ist, so sollte dem Benutzer doch bewußt bleiben, daß solche Gruppierung nicht rein aufgeht, sondern manche Doppelform [...] zu zwei oder gar drei Gruppen gehört.

\section{Zur Komplementarität von Wörterteil und Regelteil}

\subsection{Empirische Untersuchungen zu den Verweisen}

Das Problem der wechselseitigen Verzahnung von Regelteil und Wörterteil kann von beiden Seiten aus betrachtet werden. Im Folgenden soll es aus der Sicht des Wörterteils besprochen werden, und zwar zunächst des einzelnen Lexems, wie es im Wörterverzeichnis steht. Als Beispiele nehmen wir dabei die Stichwörter, die im Ergebnis des oben geschilderten Auswahlprozesses in den alphabetischen Abschnitt los... aufgenommen wurden.

Wir beginnen mit den Laut-Buchstaben-Zuordnungen, wobei die grundlegenden Zuordnungen von den besonderen und diese wiederum von den speziellen bei Fremdwörtern zu unterscheiden sind, jeweils getrennt nach Vokalen und Konsonanten. Daraus können sich - nehmen wir den «Normalfall» - so viele Regeln ergeben, wie das Wort Laute hat. Bei den Laut-Buchstaben-Zuordnungen handelt es sich dabei um die Anwendung des «phonematischen Prinzips» im Rahmen der «orthographischen Prinzipien» (vgl. Nerius et al. 1989: 68ff.).

Gehen wir beispielsweise von der phonetischen Notierung [lo:s] aus, so handelt es sich bei [1] um eine grundlegende Laut-Buchstaben-Zuordnung, wie sie in $\$ 22$ des Regelteils festgehalten ist. Für den Langvokal [o:] ermitteln wir in $\S 1$ ebenfalls eine grundlegende Laut-Buchstaben-Zuordnung (zuvor haben wir uns überzeugt, daß die «besondere Kennzeichnung der langen Vokale», wie sie die $\$ \S 6$ bis 12 enthalten, auf den vorliegenden Fall nicht zutrifft). Dagegen ergibt sich die Schreibung des Lautes [s] aus einer «besonderen» Laut-Buchstaben-Zuordnung, nämlich aus $\S 23$, der im Falle der sog. «Auslautverhärtung» gilt. Die betreffende Regel lautet: 
Die in großen Teilen des deutschen Sprachgebiets auftretende Verhärtung der Konsonanten $[b],[d],[g]$ und $[z]$ am Silbenende sowie vor anderen Konsonanten innerhalb der Silbe wird in der Schreibung nicht berücksichtigt.

Als Erläuterung «E» ist hinzugefügt:

Bei vielen Wörtern kann die Schreibung aus der Aussprache erweiterter Formen oder verwandter Wörter abgeleitet werden, in denen der betreffende Konsonant am Silbenanfang steht $[\ldots]$

Bildet man von [lo:s] eine erweiterte Form, so zeigt sich der stimmhafte Charakter des nunmehr silbenanlautenden Konsonanten: [lo:zə]. Daraus ergibt sich die Schreibung $s$ für alle Formen des Wortes, das damit dem «morphematischen Prinzip» (auch «Stammschreibung» oder «Schemakonstanz» genannt) folgt.

Analysieren wir die nunmehr ermittelte graphische Form los in syntagmatischer Hinsicht, d. h. setzen wir sie zu benachbarten und aufeinander bezogenen Formen in Beziehung, so bewegen wir uns im Bereich der Regeln für die Getrennt- und Zusammenschreibung. Im Regelteil wird davon ausgegangen, daß die getrennte Schreibung der Wörter der Normalfall und daher allein die Zusammenschreibung regelungsbedürftig ist. Die Gliederung der Regeln folgt den Wortarten, da bei den verschiedenen Wortarten spezifische Bedingungen zu beachten sind. Deshalb finden sich Regeln für die Zusammenschreibung beim Verb, beim Adjektiv und Partizip, beim Substantiv sowie bei anderen Wortarten. Lassen wir zu der Form los das Verb binden treten, so gilt $\S 34$, der lautet:

Partikeln, Adjektive oder Substantive können mit Verben trennbare Zusammensetzungen bilden. Man schreibt sie nur im Infinitiv, im Partizip I und im Partizip II sowie im Nebensatz bei Endstellung des Verbs zusammen.

Genauer trifft hier der Unterpunkt $\S 34$ (1) zu, der sich speziell auf Partikeln + Verb bezieht, wobei die ersten Bestandteile aufgezählt werden. In dieser Aufzählung, die mit $a b$-, an-, auf-, aus-, bei-beginnt, ist auch los-genannt. Anstelle von binden könnten auch andere Verben wie brechen, fahren, gehen treten. Einen Sonderfall stellt die Verbindung mit dem Verb sein dar, der durch den $§ 35$ geregelt wird. Er lautet:

Verbindungen mit sein gelten nicht als Zusammensetzung. Dementsprechend schreibt man stets getrennt.

Unter den Beispielen erscheint hinter da sein, fertig sein, inne sein auch los sein.

Gehen wir bei der syntagmatischen Analyse nun zu den Adjektiven und lassen zu los als linke Partner Morpheme wie frucht-, macht-, ziel- treten, so hätten wir in $\S 36$ des Regelteils, der die Zusammenschreibung bei Adjektiven und Partizipien regelt, neben der allgemeinen Formulierung gerne ein Beispiel gefunden, das sich auf den Wortbildungstyp mit -los (fruchtlos, machtlos, ziellos) bezieht. Leider ist dieser Fall in den Unterpunkten $\S 36$ (1) bis (6) nicht erfaßt. Wir stoßen hier also auf eine Lücke nicht nur im Wörterverzeichnis, sondern auch im Regelteil.

Einen Sonderfall in syntagmatischer Hinsicht stellt die Schreibung mit Bindestrich dar. Im Regelteil heißt es dazu:

Der Bindestrich bietet dem Schreibenden die Möglichkeit, anstelle der sonst bei Zusammensetzungen und Ableitungen üblichen Zusammenschreibung die einzelnen Bestandteile als solche zu kennzeichnen, sie gegeneinander abzusetzen und sie dadurch für den Lesenden hervorzuheben. 
Unterschieden wird dabei zwischen Regeln, in denen der Bindestrich gesetzt werden muß ( $\S \S 40-44 ; \S \S 46$ und 48-50; sog. «obligatorische Regeln»), und solchen, in denen der Gebrauch des Bindestrichs dem Schreibenden freigestellt ist ( $45, \S 51-52$; sog. "fakultative Regeln»). Will der Schreibende in einem normalerweise zusammengeschriebenen Wort die ursprüngliche Bedeutung der Bestandteile hervorheben, soll das Wort «delexikalisiert» werden, so bietet sich die Schreibung mit Bindestrich an: frucht-los (z. B. in dem Satz Dieser Baum ist frucht-los). Hier kann er sich auf $\S 45(1)$ berufen, der Beispiele wie die HochZeit, das Nach-Denken, Vor-Sätze, be-greifen bietet.

Gehen wir von der syntagmatischen zur paradigmatischen Behandlung der der phonetischen Notierung [lo:s] entsprechenden graphischen Form über, läßt sich von der Semantik und der mit ihr verbundenen Kategorisierung nicht länger absehen. Wir begeben uns damit in den Regelteil "Groß- und Kleinschreibung", soweit er sich auf bestimmte Wörter und Wortgruppen bezieht, wobei Regeln für Substantive und Desubstantivierungen, für Substantivierungen, für Eigennamen mit ihren nichtsubstantivischen Bestandteilen sowie Ableitungen von Eigennamen, des weiteren feste Verbindungen aus Adjektiv und Substantiv und schließlich Anredepronomen und Anreden zu unterscheiden sind.

Ist die graphische Form für ein Wort mit der Bedeutung (ab) zu ermitteln, in unserem Falle die Schreibung für das Adverb los, so ist der Anfangsbuchstabe selbstverständlich eine Minuskel (vorausgesetzt ist dabei, daß es sich nicht um das erste Wort einer Überschrift, eines Werktitels, eines Ganzsatzes etc. handelt). Dieser Sachverhalt ist so selbstverständlich, daß der Regelteil dafür keine spezielle Regel enthält; er ist e negativo zu folgern: Indem das Adverb zu keiner der oben genannten Kategorien mit großen Anfangsbuchstaben gehört, gilt Kleinschreibung. Regelungsbedürftig ist nur die Großschreibung und der - allerdings nicht schmale - Übergangsbereich zwischen Groß- und Kleinschreibung.

Um bei unserem Beispiel zu bleiben: Gilt es, die graphische Form für ein Wort mit der Bedeutung «Schicksal, Lotterieanteil` zu klären, also in unserem Falle die des Substantivs Los, so ist der Verweis auf $\S 55$ ( Substantive schreibt man groß») erforderlich. Handelt es sich dagegen um die graphische Form für einen mit vorangestelltem Artikel versehenen Ausruf, der zu einer sofortigen Handlung auffordert, besonders zum Gehen (z. B. in dem Satz Das ständige Los! Los! fiel ihnen auf die Nerven), liegt also eine Substantivierung vor, so ist auf $\S 57 \mathrm{zu}$ verweisen, der lautet:

Wörter anderer Wortarten schreibt man groß, wenn sie als Substantive gebraucht werden (= Substantivierungen).

In den Unterpunkten (1) bis (5) dieses Paragraphen sind dann die jeweiligen Wortarten aufgeführt, die substantiviert werden können. Im vorliegenden Falle können wir uns auf $\S 57$ (5) beziehen, der substantivierte Adverbien, Präpositionen, Konjunktionen, Interjektionen behandelt. Dort finden sich solche Beispiele wie Mich störte das ewige Hin und Her. Sein ständiges Aber stört mich. Ein vielstimmiges Ah ertönte.

Erneuter Wechsel des Aspekts: Nehmen wir die graphische Wortform als gegeben, so kann sich beim Schreiben am Zeilenende die Notwendigkeit ergeben, diese Wortform zu trennen. Auf dieses Problemfeld geht der Regelbereich 
«Worttrennung am Zeilenende» ein, wobei als Grundregel das sogenannte «syllabische Prinzip» gilt, das in $\$ 107$ folgendermaßen formuliert ist:

Geschriebene Wörter trennt man am Zeilenende so, wie sie sich bei langsamem Sprechen in Silben zerlegen lassen.

Daraus ergibt sich, daß man nur Wörter mit mehr als einer Silbe am Ende einer Zeile trennen kann. Gehen wir unsere obigen Beispiele durch, so trifft Mehrsilbigkeit z. B. zu auf die Formen lo-se, lö-sen und Lo-sung. Falls sich für diese Wörter eine Trennung am Zeilenende erforderlich macht, ist § 108 anzuführen:

Steht in einfachen Wörtern zwischen Vokalbuchstaben ein einzelner Konsonantenbuchstabe, so kommt er bei der Trennung auf die neue Zeile. Stehen mehrere Konsonantenbuchstaben dazwischen, so kommt nur der letzte auf die neue Zeile.

Nur teilweise anwendbar wäre $\S 108$ auf die Form losbinden (es handelt sich hier nicht um ein «einfaches» Wort, sondern um ein Wort mit Präfix). Hier tritt ansonsten $\S 111$ in Kraft, dem das sogenannte «morphematische Prinzip» zugrunde liegt:

Zusammensetzungen und Wörter mit Präfix trennt man zwischen den einzelnen Bestandteilen.

Die Worttrennung losbin-den kann sich also auf $\S 108$, die Trennung los-binden auf $\S 111$ berufen.

Noch einmal zurück zu den Laut-Buchstaben-Zuordnungen: Betrachtet man [lœ $\left.\int \mathrm{n}\right]$, so tritt in der phonetischen Notierung an dritter Stelle ein Laut auf, der gemäß $\S 22$ im Deutschen durch eine Buchstabenverbindung, nämlich das Trigraph sch (in löschen), wiedergegeben wird. In bestimmten Positionen entspricht dem Laut $[\delta]$ aber ein einfaches $s$ in der Schreibung. Hier handelt es sich wieder um eine «besondere» Laut-Buchstaben-Zuordnung, die in $\$ 27$ folgendermaßen festgehalten ist:

Für den Laut [ $\left.\int\right]$ am Anfang des Wortstammes vor folgendem [p] oder [t] schreibt man $s$ statt sch.

Diese Regel trifft zu auf Wörter wie spielen, verspotten; starren, Stelle, Stunde.

Für das Wort $L \ddot{\beta} \beta$ läßt die geltende Norm Aussprachevarianten zu, einmal mit kurzem offenem, einmal mit langem geschlossenem Vokal: [lœs, lø:s] (Stötzer et al., Großes Wörterbuch der deutschen Aussprache 1982: 383; Duden, Wörterbuch der deutschen Standardausssprache 1990: 474). Während sich diese Varianten in der bisherigen Rechtschreibung graphisch nicht auswirkten (zumindest nicht im Nom. Sg., wohl aber im Genitiv Sg. und im Pl.), wird in der reformierten Orthographie der lautliche Unterschied graphisch widergespiegelt. Nach § 2 des Regelteils wird die Kürze des Vokals durch Verdoppelung des folgenden Konsonantenbuchstabens gekennzeichnet, also Löss ebenso wie Hass. Dagegen schreibt man entsprechend \& 25 nach langem Vokal und Diphthong $\beta$, also $L o ̈ \beta$.

Fassen wir jetzt zusammen, auf Grund welcher Regeln sich die Schreibung jedes der hier besprochenen Wörter bestimmen läßt, so ergibt sich - getrennt nach Regelbereichen - folgende Tabelle: 


\begin{tabular}{|c|c|c|c|c|c|}
\hline \multicolumn{2}{|c|}{ Wörterteil } & \multicolumn{4}{|c|}{ Regelteil mit Regelbereichen } \\
\hline $\begin{array}{l}\text { phonet. } \\
\text { Notierg. }\end{array}$ & $\begin{array}{l}\text { graph. } \\
\text { Form }\end{array}$ & LBZ § & GZS/BS \& & GKS $\S$ & WT $\S$ \\
\hline [lo:s] & $\operatorname{los}$ & $22,1,23 \mathrm{E} 1$ & los sein 35 & $\begin{array}{l}\text { das Los! } \\
57(5)\end{array}$ & \\
\hline [lo:s] & Los & $22,1,23 \mathrm{E} 1$ & & 55 & \\
\hline [lo:s] & los- & $22,1,23 \mathrm{E} 1$ & $\begin{array}{l}\text { losubinden } \\
34(1)\end{array}$ & & 108,111 \\
\hline [lo:s] & $-\operatorname{los}$ & $22,1,23 \mathrm{E} 1$ & $\begin{array}{l}\text { fruchtlos } \\
\text { frucht-los } \\
45(1)\end{array}$ & & $\begin{array}{l}111 \\
\text { F } 0(2)\end{array}$ \\
\hline$\left[\operatorname{lœ} \int \mathrm{n}\right]$ & löschen & $22,1,22 \ldots$ & & & 109 \\
\hline [lo:zə] & lose & $22,1,22$ & lose sein 35 & & 108 \\
\hline [lø:zn] & lösen & $22,1,22 \ldots$ & & & 108 \\
\hline [lœes] & Löss & $22,1,2$ & & 55 & \\
\hline$[1 \varnothing: s]$ & Löß & $22,1,25$ & & 55 & \\
\hline [lo:zun] & Losung & $22,1,22,1,22$ & & 55 & 108 \\
\hline
\end{tabular}

Abkürzungen für die Regelbereiche:

LBZ: Laut-Buchstaben-Zuordnungen

GZS: Getrennt- und Zusammenschreibung

BS: Schreibung mit Bindestrich

GKS: Groß- und Kleinschreibung

WT: Worttrennung am Zeilenende

\subsection{Grundsätzliches zu den Verweisen vom Wörterteil in den Regelteil}

Der Blick auf die Tabelle zeigt die hohe Redundanz eines Verfahrens, das bei jedem Stichwort auf sämtliche zugrunde liegenden Regeln verweist. Es kehren die Paragraphennummern, die sich auf die grundlegenden Regeln beziehen ( $\S 1$ und $\S 22$ bei den Laut-Buchstaben-Zuordnungen, $\S 55$ bei der Großschreibung der Substantive, $\S 108$ und $\S 111$ bei der Worttrennung am Zeilenende) ständig wieder. Das eigentlich Interessante an diesem Verfahren sind die Verweise auf die «besonderen» Regeln. Interessant wird es für den Linguisten aber auch, wenn auf diese Weise "Lücken" im Regelteil benannt werden. Wichtig ist, daß diese «Lücken» den Autoren des Regelteils bewußt sind, waren sie doch im Interesse einer übersichtlichen Darstellung des Regelteils, der als Grundlage für die Praxis amtliche Geltung erlangen sollte, gezwungen, bestimmte «Selbstverständlichkeiten» auszusparen. Dazu ist z. B. eine Regel für die Kleinschreibung von eindeutigen Nichtsubstantiven zu rechnen.

Aber nicht in jedem Falle kann man sicher sein, daß Regeln bewußt ausgespart wurden. Im Regelbereich Laut-Buchstaben-Zuordnungen $\S 4$ heißt es z. B.:

In acht Fallgruppen verdoppelt man den Buchstaben für den einzelnen Konsonanten nicht, obwohl dieser einem kurzen betonten Vokal folgt.

Wurde hier der Fall, daß sch nach kurzem betonten Vokal wie in löschen nicht verdoppelt wird, bewußt weggelassen? 
Oder im Regelbereich Getrennt- und Zusammenschreibung § 36 (2), der die Zusammensetzungen betrifft, «bei denen der erste oder zweite Bestandteil in dieser Form nicht selbständig vorkommt»: Wurden hier Beispiele für die Zusammensetzungen mit -los, die an der Grenze zu den Ableitungen stehen (fruchtlos, machtlos, ziellos), absichtlich nicht aufgeführt?

Oder im Regelbereich Schreibung mit Bindestrich $\S 45$ (1), der die Hervorhebung einzelner Bestandteile betrifft. Man vermißt hier ein Beispiel für BindestrichSchreibungen bei delexikalisierten Adjektiven wie frucht-los. Oder ist in diesem Falle auch die Schreibung Frucht-los zulässig? An diesem Punkt ist der Bereich dessen, was amtlich geregelt werden kann und sollte, offensichtlich überschritten.

Bei der Bearbeitung des Wörterverzeichnisses mußte unter den theoretisch möglichen Verweisen ausgewählt werden. Dabei wurde hinsichtlich der einzelnen Regelbereiche unterschiedlich verfahren.

Auf den Regelbereich der Laut-Buchstaben-Zuordnungen wurde generell nicht verwiesen. Alleinige Ausnahme: Verweise auf $\S 9 \mathrm{E} 2$, der Wörter mit aa und $o o$ betrifft:

Bei Umlaut schreibt man nur $\ddot{a}$ bzw. $\ddot{o}$, zum Beispiel:

Härchen - aber: Haar; Pärchen - aber: Paar; Säle - aber: Saal; Bötchen - aber: Boot

Dagegen enthält das Wörterverzeichnis viele Verweise auf den Regelbereich der Getrennt- und Zusammenschreibung (§§33-39). So verweist z. B. §33 (1) auf die Regel für die Zusammenschreibung bei Zusammensetzungen, die aus Substantiv + Verb gebildet sind, wozu folgende Beispiele gehören:

brandmarken (gebrandmarkt, zu brandmarken), handhaben, lobpreisen, maßregeln, nachtwandeln, schlafwandeln, schlussfolgern, wehklagen, wetteifern

Im Wörterverzeichnis findet sich an alphabetischer Stelle dann z. B. folgende Eintragung:

wettueifern, ...laufen, ....rennen $\S 33(1) ; \ldots$ machen $\S 34(3)$

Der Grund dafür, daß bei dem vierten Beispiel, nämlich wettmachen, auf §34(3) verwiesen wird, ist der, daß man dieses Verb nur im Infinitiv, im Partizip II sowie im Nebensatz bei Endstellung des Verbs zusammenschreibt, während wetteifern, wettlaufen und wettrennen stets zusammengeschrieben werden.

Auf den Regelbereich «Schreibung mit Bindestrich» wird ebenfalls in einer gröBeren Zahl von Fällen verwiesen, aber nur auf die $\$ \S 40-45$, die Zusammensetzungen und Ableitungen betreffen, die keine Eigennamen als Bestandteile enthalten. Da die $\$ \S 46-52$ sich auf Zusammensetzungen und Ableitungen beziehen, die Eigennamen enthalten, diese aber in das Wörterverzeichnis grundsätzlich nicht aufgenommen wurden, treten diese Paragraphen in den Verweisen nicht auf.

Viele Verweise betreffen den Regelbereich «Groß- und Kleinschreibung», allerdings ohne die $\$ \S 53$ und 54 , die der Kennzeichnung des Anfangs bestimmter Texteinheiten durch Großschreibung gelten. Die Verweise beziehen sich auf die Großschreibung der Substantive (\$55), auf die Kleinschreibung von Desubstantivierungen ( $\$ 56$ ), auf die Großschreibung von Substantivierungen ( $\$ 57$ ), auf die Kleinschreibung von Adjektiven, Partizipien und Pronomen, die formale Merkmale von Substantivierungen aufweisen ( $\$ 58)$. Obwohl Eigennamen und ihre Ableitungen ( §59-62) grundsätzlich nicht in das Wörterverzeichnis aufgenom- 
men wurden, gibt es auf den $\$ 60$, der die Schreibung nichtsubstantivischer Bestandteile in mehrteiligen Eigennamen (z. B. Stiller Ozean) regelt, einzelne Verweise. Weitere Verweise betreffen die Groß- bzw. Kleinschreibung in festen Verbindungen aus Adjektiv und Substantiv ( $\$$ 63-64) sowie Anredepronomen und Anreden ( $\$ 85-66)$.

Auf den Regelbereich «Zeichensetzung» finden sich im Wörterverzeichnis fast keinerlei Verweise, was nicht so selbstverständlich ist, wie es auf den ersten Blick scheint. Baudusch (1991) hat darauf aufmerksam gemacht, daß es in Rechtschreibwörterbüchern eine nicht geringe Zahl von Stichwörtern gibt (z. B. Konjunktionen), die für die Zeichensetzung bedeutsam sind und sich für die Verzahnung von Wörterteil und Regelteil anbieten. In der vorliegenden Bearbeitung des amtlichen Wörterverzeichnisses gibt es nur wenige Verweise auf den $\S 102$, der die Schreibung von Abkürzungen und Kurzwörtern behandelt (z. B. $A B C$-Waffen), und auf den $\S 106$, der die Schreibung mit Schrägstrich (z. B. September/Oktober-Heft) regelt.

Verweise auf den Regelbereich «Worttrennung am Zeilenende» sind grundsätzlich nicht gegeben worden, obwohl sich die Verzahnung von Regel- und Wörterteil auch hier anbieten würde.

\subsection{Das Wörterverzeichnis als Register für die Beispiele des Regelteils}

Beabsichtigt war ursprünglich, auf alle Lexeme, die in den verschiedenen Regelbereichen (mit Ausnahme der Zeichensetzung) als Beispiele auftreten, im Wörterverzeichnis einen besonders gekennzeichneten Verweis (etwa durch Unterstreichen) anzubringen. Diese Verweise, die vor allem für den eiligen Benutzer eine Hilfe sind, würden insgesamt zur besseren Verzahnung von Regel- und Wörterteil beitragen. Leider mußte bei der Drucklegung aus Zeitgründen auf solche Verweise vorläufig verzichtet werden. Die dazu bereits bestehenden Vorarbeiten sollen ergänzt und später publiziert werden.

\section{Das Orthographikon als Aufgabe}

Anknüpfend an die Untersuchungen und Erörterungen zur Komplementarität von Regelteil und Wörterteil der amtlichen Deutschen Rechtschreibung (Tübingen 1996), die in der Darbietung von Regeln und Wortschatz stark die Bedürfnisse der Schulen und Behörden berücksichtigt, stellt sich für den Sprachwissenschaftler die Frage, wie ein Nachschlagewerk gestaltet sein müßte, für das vorwiegend linguistische Gesichtspunkte maßgebend sind. Für ein solches Nachschlagewerk sind die Begriffe «Rechtschreibwörterbuch» oder «Orthographiewörterbuch» wenig geeignet, da hier bereits in der Benennung die Dominanz der Wortschreibung zum Ausdruck kommt. Für den Linguisten geht es um eine Kodifizierung der Normen der graphischen Ebene des Sprachsystems, wobei an ein Mehrebenenmodell mit hierarchisch gegliederten bi- bzw. trilateralen Ebenen als Teilsystemen gedacht ist. Der Wissensspeicher, der genau diese Aufgabe übernehmen sollte, ist in den letzten Jahren als "Orthographikon" in die sprachwissenschaftliche Diskussion eingeführt worden (vgl. Scharnhorst 1995). Da die Normen der 
graphischen Ebene sowohl aus generellen als auch aus «singulären Regeln〉 bestehen (zu diesem Begriff vgl. Kohrt 1987), liegt das wesentliche innere Merkmal des Orthographikons darin, daß generelle und singuläre Regeln wechselseitig aufeinander bezogen sind, also der Grundsatz der Komplementarität verwirklicht wird.

Beim Orthographikon kann man drei Kodifizierungsstufen unterscheiden: Das kleine, das mittlere und das große Orthographikon. Während das kleine Orthographikon nur die grundlegenden Generalisierungen und das entsprechende Inventar der Elemente der graphischen Ebene enthält, kodifiziert das mittlere Orthographikon neben den grundlegenden Generalisierungen auch die hierarchisch untergeordneten. Komplementär dazu gibt es wieder die entsprechenden Inventare von Elementen. Das große Orthographikon umfaßt neben den grundlegenden und den hierarchisch untergeordneten auch Regeln von geringem Verallgemeinerungsgrad sowie die entsprechenden Inventare. In den Termini des Konzepts von Zentrum und Peripherie kann man sagen, daß das kleine Orthographikon nur das Zentrum der graphischen Ebene darstellt; hingegen beschreibt das mittlere Orthographikon außer dem Zentrum auch die Übergangssphäre, und das große Orthographikon umfaßt neben Zentrum und Übergangssphäre zusätzlich die Peripherie.

Damit ist ein Forschungsfeld angedeutet, das sowohl für die sprachwissenschaftliche Grundlagenforschung als auch für die praxisorientierte Forschung große Bedeutung hat.

\section{Literatur}

Augst, Gerhard (1975): Lexikon zur Wortbildung. Morpheminventar. 3 Bände. Tübingen. (= Forschungsberichte des Instituts für deutsche Sprache 24)

Augst, Gerhard / Schaeder, Burkhard (Hrsg.) (1991): Rechtschreibwörterbücher in der Diskussion. Geschichte - Analyse - Perspektiven. Frankfurt a. M. / Bern / New York / Paris. (= Theorie und Vermittlung der Sprache 13)

Baudusch, Renate (1991): Zur doppelten Kodifikation der Interpunktion im Rechtschreibwörterbuch. In: Gerhard Augst / Burkhard Schaeder (Hrsg.): Rechtschreibwörterbücher in der Diskussion. Geschichte - Analyse - Perspektiven. Frankfurt a. M./Bern/New York/Paris, 383-402. (= Theorie und Vermittlung der Sprache 13)

Daneš, František (1982): Zur Theorie des sprachlichen Zeichensystems. In: Jürgen Scharnhorst / Erika Ising in Zusammenarbeit mit Karel Horálek und Jaroslav Kuchař (Hrsg.): Grundlagen der Sprachkultur. Beiträge der Prager Linguistik zur Sprachtheorie und Sprachpflege. 2. Band, Berlin, 132-173. (= Sprache und Gesellschaft 8)

Duden (1902): Orthographisches Wörterbuch der deutschen Sprache. Nach den für Deutschland, Österreich und die Schweiz gültigen amtlichen Regeln. Siebente Auflage, Leipzig/Wien.

Duden (1989): Deutsches Universalwörterbuch. 2., völlig neu bearbeitete und stark erweiterte Auflage. Hrsg. von Günther Drosdowski. Mannheim/Wien/Zürich.

Duden (1990): Wörterbuch der deutschen Standardaussprache. 3., völlig neu bearbeitete und erweiterte Auflage. Mannheim/Wien/Zürich.

Duden (1991): Rechtschreibung der deutschen Sprache. 20., völlig neu bearbeitete und erweiterte Auflage. Mannheim/Leipzig/Wien/Zürich. 
Filipec, Josef (1966): Probleme des Sprachzentrums und der Sprachperipherie im System des Wortschatzes. In: Travaux Linguistiques de Prague 2. Les problèmes du centre et de la périphérie du système de la langue, 257-275.

Gabler, Birgit (1992): Orthographische Varianten in ausgewählten Auflagen des Dudens seit 1880. In: Dieter Nerius / Jürgen Scharnhorst (Hrsg.): Studien zur Geschichte der deutschen Orthographie. Hildesheim/Zürich/New York, 367-397. (= Germanistische Linguistik 108-109)

Grimm, Jacob / Grimm, Wilhelm (1854-1960): Deutsches Wörterbuch. Leipzig.

Heller, Klaus (1970): Der Wortschatz unter dem Aspekt des Fachwortes - Versuch einer Systematik. In: Wissenschaftliche Zeitschrift der Karl-Marx-Universität Leipzig, Gesellschaftsund Sprachwissenschaftliche Reihe, 19/4, 531-544. [Wieder abgedruckt in: Walther v. Hahn (Hrsg.) (1981): Fachsprachen. Darmstadt, 218-238. (= Wege der Forschung 498)]

Heller, Klaus (1980): Untersuchungen zur Begriffsbestimmung des Fremdwortes und zu seiner Schreibung in der deutschen Gegenwartssprache. Phil. Diss., Leipzig.

Heller, Klaus / Scharnhorst, Jürgen (1983): Zu den Begriffen «Zentrum» und «Peripherie». In: Linguistische Studien, Reihe A, 113/II, Berlin, 78-93.

Heller, Klaus / Walz, Brigitte (1992): Zur Geschichte der Fremdwortschreibung im Deutschen. Beobachtungen von Campe bis Duden. In: Dieter Nerius / Jürgen Scharnhorst (Hrsg.): Studien zur Geschichte der deutschen Orthographie. Hildesheim/Zürich/New York, 277-338. (= Germanistische Linguistik 108-109)

Herberg, Dieter (1993): Deutsche Rechtschreibwörterbücher - wie sie sind und wie sie sein sollten. In: Zeitschrift für Germanistische Linguistik, 21, 334-353.

Internationaler Arbeitskreis für Orthographie (Hrsg.) (1996): Deutsche Rechtschreibung. Regeln und Wörterverzeichnis. Amtliche Regelung. Tübingen.

Klappenbach, Ruth / Steinitz, Wolfgang (Hrsg.) (1964-1977): Wörterbuch der deutschen Gegenwartssprache. 6 Bände, Berlin.

Kohrt, Manfred (1987): Theoretische Aspekte der deutschen Orthographie. Tübingen. (= RGL 70)

Mentrup, Wolfgang (1991): Zum Wörterverzeichnis als Bestandteil des Orthographiebuches und $z$ u seiner Gestaltung. Thesenpapier mit Beispielen. [Vervielfältigtes Manuskript, 12 Seiten und 6 Anlagen.]

Mentrup, Wolfgang (1992): Wörterverzeichnis. In: Internationaler Arbeitskreis für Orthographie (Hrsg.): Deutsche Rechtschreibung. Vorschläge zu ihrer Neuregelung. Tübingen, 209213.

Muthmann, Gustav (1994): Doppelformen in der deutschen Sprache der Gegenwart. Studie zu den Varianten in Aussprache, Schreibung, Wortbildung und Flexion. Tübingen. (= RGL 145)

Nerius, Dieter / Scharnhorst, Jürgen (1980): Grundpositionen der Orthographie. In: Dieter Nerius I Jürgen Scharnhorst (Hrsg.): Theoretische Probleme der deutschen Orthographie. Berlin, 11-73. (= Sprache und Gesellschaft 16)

Nerius, Dieter et al. (1989): Deutsche Orthographie. 2., durchgesehene Auflage, Leipzig.

Nerius, Dieter / Scharnhorst, Jürgen (Hrsg.) (1992): Studien zur Geschichte der deutschen Orthographie. Hildesheim/Zürich/New York. (= Germanistische Linguistik 108-109)

Österreichisches Wörterbuch (1990): 37., überarbeitete Auflage, Wien.

Pfeifer, Wolfgang (1995): Etymologisches Wörterbuch des Deutschen. München.

Regeln (1880): Regeln und Wörterverzeichnis für die deutsche Rechtschreibung zum Gebrauch in den preußischen Schulen. Hrsg. im Auftrage des Königlichen Ministeriums der geistlichen, Unterrichts- und Medizinal-Angelegenheiten. Berlin. [Erneut als Sammlung Duden 2] 
Regeln (1902): Regeln für die deutsche Rechtschreibung nebst Wörterverzeichnis. Hrsg. im Auftrage des Königlich Preußischen Ministeriums der geistlichen, Unterrichts- und Medizinal-Angelegenheiten. Berlin. [Erneut als Sammlung Duden 4]

Scharnhorst, Jürgen /Ising, Erika in Zusammenarbeit mit Karel Horálek und Jaroslav Kuchař (Hrsg.) (1976/1982): Grundlagen der Sprachkultur. Beiträge der Prager Linguistik zur Sprachtheorie und Sprachpflege. 2 Bände. Berlin. (= Sprache und Gesellschaft 8)

Scharnhorst, Jürgen (1991): Das Orthographiewörterbuch unter dem Aspekt von Zentrum und Peripherie. In: Gerhard Augst / Burkhard Schaeder (Hrsg.): Rechtschreibwörterbücher in der Diskussion. Geschichte - Analyse - Perspektiven. Frankfurt a. M./Bern/New York/Paris, 491-507. (= Theorie und Vermittlung der Sprache 13)

Scharnhorst, Jürgen (1993): Der Wortschatz unter dem Aspekt von Zentrum und Peripherie. In: Gerhard Bartels / Inge Pohl (Hrsg.): Wortschatz - Satz - Text. Beiträge der Konferenzen in Greifswald und Neubrandenburg 1992. Frankfurt a. M./Berlin/Bern/New York/Paris, 63-76.

Scharnhorst, Jürgen (1995): Das Orthographikon. In: Petra Ewald / Karl-Ernst Sommerfeldt (Hrsg.): Beiträge zur Schriftlinguistik. Frankfurt a. M./Bern/New York/Paris, 291-310.

Stötzer, Ursula et al. (1982): Großes Wörterbuch der deutschen Aussprache. Leipzig.

Travaux (1966): Travaux linguistiques de Prague 2. Les problèmes du centre et de la périphérie du système de la langue. Prague.

Wiegand, Herbert Ernst (1989a): Aspekte der Makrostruktur im allgemeinen einsprachigen Wörterbuch: alphabetische Anordnungsformen und ihre Probleme. In: Franz Josef Hausmann, Oskar Reichmann, Herbert Ernst Wiegand, Ladislav Zgusta (Hrsg.): Wörterbücher. Erster Teilband. Berlin/New York, 371-409.

Wiegand, Herbert Ernst (1989b): Der Begriff Mikrostruktur: Geschichte, Probleme, Perspektiven. In: Franz Josef Hausmann, Oskar Reichmann, Herbert Ernst Wiegand, Ladislav Zgusta (Hrsg.): Wörterbücher. Erster Teilband. Berlin/New York, 409-462. 